\title{
Eosinophilic Esophagitis Without Abundant Eosinophils? The Expanding Spectrum of a Disease That Is Difficult to Define
}

\author{
Leona A. Doyle $\cdot$ Robert D. Odze
}

Published online: 30 April 2011

(C) Springer Science+Business Media, LLC 2011

In 2007, the American Gastroenterological Association (AGA) provided a systematic review and consensus recommendations for the diagnosis and treatment of eosinophilic esophagitis (EoE) [1]. At that time, EoE was defined as a distinct clinical and pathologic entity characterized by the presence of clinical symptoms of esophageal dysfunction, an absence of gastroesophageal reflux disease (GERD) by either lack of response to proton pump inhibitor (PPI) therapy or normal $\mathrm{pH}$ monitoring values of the distal esophagus, and documentation of $\geq 15$ eosinophils per high power field (Eos/HPF) in mucosal biopsies of the esophagus. Since its original description in children, EoE is now recognized as a disorder of adults as well. EoE is believed to represent an immune mediated disease for a variety reasons, but primarily because of its known association with other hypersensitivity or allergic disorders, the beneficial effect to steroid therapy in many patients, and by an abundance of studies that have shown an association with T-helper 2 mediated immunity via the action of eotaxin-3 and cytokines IL-13 and IL-5 [2-4]. Although EoE likely represents an allergic disorder, ultimately, the underlying initiating and perpetuating pathogenetic mechanisms remain unknown, which is partly the reason why it is difficult to establish definitive clinical and pathologic diagnostic criteria. In fact, the number of patients diagnosed with EoE is steadily increasing, and although this may be partly due to increasing awareness of this entity among clinicians, unfortunately, due to the lack of standardized diagnostic criteria prior to 2007, interpretation

L. A. Doyle · R. D. Odze $(\bowtie)$

Department of Pathology, Brigham and Women's Hospital,

Harvard Medical School, 75 Francis Street, Boston,

MA 02115, USA

e-mail: rodze@partners.org and comparison of data from prior studies (pre 2007) is difficult.

There is a growing body of data, mainly related to the pathology, pathogenesis, natural history, and effect of certain forms of treatment, to suggest that the diagnostic criteria put forth in 2007 for EoE was in need of revision. For instance, there is evidence to suggest that there is a potential pathogenetic (or synergistic) relationship between EoE and GERD, as exemplified by the recent identification of patients who have overlapping clinical and pathologic features. Several studies have shown that eosinophil counts in patients with GERD may reach, or even exceed, the levels often associated with EoE [5-8]. Conversely, it is now being increasingly recognized that some patients with clinical and endoscopic features of EoE, but without GERD, contain lower than the normal threshold numbers of peak Eos/HPF in their esophageal biopsies. Also interesting is the recent identification of a subgroup of patients with esophageal eosinophilia and with clinical/endoscopic symptoms of EoE who respond positively to PPI therapy, termed "PPI-responsive EoE." For instance, Ngo et al. described three patients with dysphagia and food impaction and $>20$ Eos/HPF in their esophageal biopsies who, following treatment with PPI, became clinically asymptomatic, achieved normal endoscopic findings, and normal, or near normal, intraepithelial eosinophil counts [9]. A recent prospective study by Molina-Infante et al. [8] of 35 patients with $\geq 15$ Eos/HPF also provided evidence that a subgroup of patients with EoE may respond to PPI therapy.

This and other clinical and pathologic data suggest that there may be a pathogenetic link between EoE and GERD, and that the original criteria used to diagnose EoE was in need of revision. As a result, the 2011 updated consensus recommendations for EoE in children and adults has altered the definition of EoE as "a disorder that represents a 
chronic/immune antigen mediated esophageal disease characterized clinically by symptoms related to esophageal dysfunction and histologically by eosinophil-predominant inflamation." The updated diagnostic guidelines emphasize that EoE is a clinicopathologic disorder, characterized by symptoms related to esophageal dysfunction and pathologically by one or more biopsies that show eosinophil predominant inflammation. Although 15 Eos/HPF is considered a minimum threshold necessary for diagnosing EoE, it is now recognized that there are a few exceptions that are possible. The diagnostic guidelines recognize an emerging body of literature that describe a subset of patients with symptoms and histopathologic findings of EoE that are responsive to PPI treatment and who may, or may not, have well-documented GERD. Until more is known regarding this subgroup of patients, it is recommended that these patients be diagnosed as "PPI-responsive eosinophilia." Future studies may help determine if PPIs help diminish the immune/antigen driven response by having anti-inflammatory action or by providing a barrier to healing.

In this issue of Digestive Diseases and Sciences, Ravi et al. [10] provide data that contribute to the proposed expansion of the original conceptual definition of EoE, by including patients who have clinical and endoscopic features characteristic of this disorder, but who have esophageal biopsies at index that show peak eosinophil counts $\leq 15$ Eos/HPF, and thus termed "low-grade eosinophilia". In their study, 59 patients with esophageal eosinophilia, ranging from 1 to 14 Eos/HPF were identified during a 5-year period between January 2002 and January 2007, from the Mayo Clinic, Rochester, in a retrospective casecontrol study. The study patients with low-grade esophageal eosinophilia were then further subdivided into two groups, the first with 1-9 Eos/HPF $(N=23)$ and the second with 10-14 Eos/HPF $(N=36)$ for the purpose of statistical and outcome analysis, although the biological reasons for the group separation were not clear. Nevertheless, the study group was compared to 418 control patients who fulfilled the "historical" definition of EoE, by showing $\geq 15$ Eos/HPF in their esophageal biopsies. Since all of the patients, both cases and controls, in this study were biopsied prior to the 2007 consensus recommendations, exclusion of GERD was not part of the diagnostic criteria. In fact, $35 \%$ and $27 \%$ of the cases and controls, respectively, had consumed PPIs prior to their initial endoscopy with biopsies. A minimum of four biopsies were obtained from $10 \mathrm{~cm}$ proximal to the lower esophageal sphincter. An additional $31 \%$ of patients had two biopsies obtained from the distal esophagus. All initial biopsies were read by a single pathologist and peak eosinophil counts (recorded per HPF) were provided. Clinically, patients in the study group revealed a significantly lower prevalence rate of asthma or allergic rhinitis $(P=0.04)$. However, presenting symptoms, including dysphagia, and endoscopic signs characteristic of EoE, such as ringed esophagus, furrows, white exudates, and non-distal strictures, were similar in the study and control groups. Dysphagia was reported in $83 \%$ and $93 \%$, and food impaction in $27 \%$ and $33 \%$, of the cases and controls, respectively. Heartburn was also a common symptom in both patient groups $(>60 \%)$. The follow-up data provided by Ravi et al. is interesting and provides the basis for their subsequent conclusions. Upon follow-up, 14 study patients had a second endoscopy with biopsies, of which five revealed $\geq 15$ Eos/HPF in their esophageal mucosal biopsies. Interestingly, three of these five patients were treated with PPIs prior to their repeat endoscopy and biopsy, but this treatment did not appear to effect the degree of esophageal eosinophilia upon follow-up. In fact, three of the five patients with $\geq 15$ Eos/HPF on repeat biopsy were treated with aerosolized fluticasone and showed clinical improvement. An additional nine study patients did not have a repeat endoscopy but were also treated with topical steroids, and six showed clinical improvement. Finally, a further 18 study patients were treated with PPI therapy, with symptomatic improvement in 11. Based on this data, the authors concluded that some patients with $<15$ Eos/ HPF in their esophageal biopsies ("low-grade esophageal eosinophilia") have a similar clinical and endoscopic phenotype to patients with $\geq 15$ Eos/HPF, the latter who fulfill the historical definition of EoE. The authors also suggest that steroid treatment may be a viable option for some patients with low-grade esophageal eosinophilia and other clinical or endoscopic findings of EoE.

Although the data provided by Ravi et al. is both interesting and provocative, there are a number of limitations to this study, many of which were openly acknowledged by the authors, that impede the ability to understand the clinical or biological significance of patients with EoEtype symptoms and low-grade esophageal eosinophilia. For instance, the study did not exclude GERD by objective means (such as $\mathrm{pH}$ and/or impedance monitoring), it was retrospective in nature, had rather short follow-up intervals in some patients, used a historical definition of EoE based solely on eosinophil counts in the control group, included patients treated with PPIs prior to their initial biopsy, had incomplete follow-up in the majority of patients, lacked determination of interobserver variability by pathologists, and lacked distal biopsies in a large percentage of patients. Finally, the study group represented a highly select group of patients (selection bias) who were concerned enough to undergo endoscopy with biopsy.

Nevertheless, it is clear that some study patients with clinical and endoscopic findings consistent with EoE responded well to topical steroids, and as such, may 
represent an early or alternative form of EoE. However, it is important to note that there are several potential reasons why a patient with EoE may have $<15$ Eos/HPF in their biopsies at one point in time. These include, but are not limited to, sampling error, inadequate number (or location) of biopsies, presence of chronic disease, response to treatment (particularly PPIs), and the difficulty of evaluating eosinophils when many such cells are degranulated. Regarding possible response to PPIs, as mentioned above, this is complicated by the currently unknown pathogenetic interplay between EoE and GERD. PPIs may have an effect on patients who clinically fulfill the diagnostic criteria of EoE for a variety of reasons, such as lack of $\mathrm{pH}$ impedance measurements in patients who have normal $\mathrm{pH}$ values obtained, or lack of patient adherence to PPI therapy. Furthermore, PPIs may act on esophageal eosinophilia via currently unknown mechanisms. Patients with EoE may have an enhanced sensitivity to acid even in the absence of pathologic reflux measured by conventional criteria.

Despite the limitations of this study, the results by Ravi et al. propose the possible existence of a subgroup of EoE patients who have low-grade esophageal eosinophilia, and, at minimum, defy the guidelines used to diagnose this disorder. Clinicians and pathologists alike need to be aware of a potential subgroup of patients who may have a form of EoE, perhaps at an earlier point in the natural history of this disorder, that may benefit from steroid therapy. In order to determine the significance of these findings in the future, this data should be validated prospectively and with the use of rigorous $\mathrm{pH}$ testing, preferably with impedance measurements as well. The ongoing investigation between the relationship of GERD and EoE will likely reveal a biological overlap between these two entities and will help elucidate the roll of acid reflux in causing EoE and vice versa.

\section{References}

1. Furuta GT, Liacouras CA, Collins MH, et al. First international gastrointestinal eosinophil research symposium (FIGERS) subcommittees. Eosinophilic esophagitis in children and adults: A systematic review and consensus recommendations for diagnosis and treatment. Gastroenterology. 2007;133:1342-1363.

2. Blanchard C, Mingler MK, Vicario M, et al. IL-13 involvement in eosinophilic esophagitis: Transcriptome analysis and reversibility with glucocorticoids. J Allergy Clin Immunol. 2007;120: 1292-1300.

3. Blanchard C, Stucke EM, Rodriguez-Jimenez B, et al. A striking local esophageal cytokine expression profile in eosinophilic esophagitis. J Allergy Clin Immunol. 2011;127:208, 217, 217. e1-7.

4. Zuo L, Fulkerson PC, Finkelman FD, et al. IL-13 induces esophageal remodeling and gene expression by an eosinophilindependent, IL-13R alpha 2-inhibited pathway. J Immunol. 2010;185:660-669.

5. Rodrigo S, Abboud G, Oh D, et al. High intraepithelial eosinophil counts in esophageal squamous epithelium are not specific for eosinophilic esophagitis in adults. Am J Gastroenterol. 2008; 103:435-442.

6. Dellon ES, Gibbs WB, Fritchie KJ, et al. Clinical, endoscopic, and histologic findings distinguish eosinophilic esophagitis from gastroesophageal reflux disease. Clin Gastroenterol Hepatol 2009;7:1305,13; quiz 1261.

7. Peterson KA, Thomas KL, Hilden K, Emerson LL, Wills JC, Fang JC. Comparison of esomeprazole to aerosolized, swallowed fluticasone for eosinophilic esophagitis. Dig Dis Sci. 2010;55: 1313-1319.

8. Molina-Infante J, Ferrando-Lamana L, Ripoll C, et al. Esophageal eosinophilic infiltration responds to proton pump inhibition in most adults. Clin Gastroenterol Hepatol. 2011;9:110-117.

9. Ngo P, Furuta GT, Antonioli DA, Fox VL. Eosinophils in the esophagus-peptic or allergic eosinophilic esophagitis? Case series of three patients with esophageal eosinophilia. Am J Gastroenterol. 2006;101:1666-1670.

10. Ravi K, Talley NJ, Smyrk TC, et al. Low grade esophageal eosinophilia in adults: An unrecognized part of the spectrum of eosinophilic esophagitis? Dig Dis Sci. (Epub ahead of print). doi: 10.1007/s10620-011-1594-1 